\title{
Epidemiology of tick-borne encephalitis in Switzerland, 2005 to 2011
}

M Schuler ${ }^{1}$, H Zimmermann², E Altpeter ${ }^{2}$, U Heininger (Ulrich.Heininger@ukbb.ch) ${ }^{1}$

1. Division of Infectious Diseases and Vaccines, University Children's Hospital (UKBB), Switzerland

2. Division of Transmissible Diseases, Federal Office of Public Health (FOPH), Berne, Switzerland

Schuler M, Zimmermann H, Altpeter E, Heininger U. Epidemiology of tick-borne encephalitis in Switzerland, 2005 to 2011. Euro Surveill. $2014 ; 19(13)$ :pii=20756. Available online: http://www.eurosurveillance.org/ViewArticle.aspx?Articleld=20756

We provide an update on the epidemiology and disease characteristics of tick-borne encephalitis (TBE) in Switzerland. Data were collected through the mandatory notification system of the Federal Office of Public Health. Between 2005 and 2011, a total of 1,055 TBE cases were reported, with a peak of 244 cases in 2006. The average yearly incidence was 2.0/100,000 inhabitants nationwide, with the highest regional value $(7.8 / 100,000)$ in eastern Switzerland. Incidence by age peaked in 60-69 year-old patients, males predominated in all age groups. Most patients suffered from meningoencephalitis $(n=567)$ or meningitis $(n=246)$, seven of 1,055 patients ( $0.7 \%$ ) died. Of 617 patients who were 40 years and older, 442 (72\%) suffered from a severe course of illness, compared to 196 (51\%) of 384 patients younger than 40 years. Most patients were not immunised against TBE, and in 33 of 1,055 patients $(3 \%)$, vaccine failure was found possible. Ongoing surveillance of TBE and intensified efforts in promoting TBE vaccination for the population at risk will be needed in light of its considerable morbidity.

\section{Introduction}

Tick-borne encephalitis (TBE) is an acute infection involving the central nervous system (CNS) with potentially serious outcome. It is caused by the TBE virus (TBEV), which belongs to the Flavivirus family. Transmission of TBEV to humans occurs by bites of infected ticks, in Europe mainly Ixodes ricinus. After an incubation period of four to 28 days (usually seven to 14 days), the clinical presentation of TBE usually starts with one to seven days of influenza-like illness (ILI), followed by an afebrile and mostly asymptomatic interval [1]. A few days later, CNS manifestations such as meningitis, meningoencephalitis or encephalomyelitis may occur in approximately one third of patients [2]. The case fatality rate is less than $2 \%$, whereas permanent sequelae such as cognitive or neuropsychiatric complaints, balance disorders, headaches, dysphasia, hearing defects, and spinal paralysis are reported in up to $46 \%$ of patients [3].
In Switzerland, TBE occurs in specified endemic areas and is a notifiable disease. The average national incidence of TBE in Switzerland between 1984 and 2004 was $1.4 / 100,000 / y e a r$, with values reaching up to 7.9 in endemic cantons (highest values: Thurgau 7.9, Schaffhausen 4.3, Zurich 2.7, and Aargau 2.2) [4]. Age distribution of patients revealed two peaks, i.e. in six to 14 year-old children and in 60 to 69 year-old adults. Children younger than six years were rarely affected [4].

Effective vaccines are available to prevent TBE, but no curative treatment exists $[2,5]$. In Switzerland, TBE vaccination coverage with at least one dose in 2007 was $17 \%$ for the whole country, with no difference between males and females [6]. The highest coverage was $25 \%$ in the 13 to 19 year-olds; In endemic regions, coverage varied between 28 and $47 \%$ [personal communication A Zacharias, 14 March 2014]. In the period 2010 to 2012, vaccination coverage in 16 year-old adolescents in endemic regions ranged from 42 to $71 \%$ for one and 39 to $64 \%$ for three doses [7]. While coverage with the first dose of TBE vaccine indicates the population with an initiated immunisation series, optimal protection requires a complete primary series with three vaccine doses, followed by regular booster doses.

The aim of this study was to provide an update on the epidemiology and disease characteristics of TBE in Switzerland for the years 2005 to 2011 in light of a notable increase in reported cases in recent years [8].

\section{Methods}

\section{Data source and collection}

TBE has been a notifiable disease in Switzerland since 1984. Laboratories have to report to the Federal Office of Public Health (FOPH) and the cantonal medical officer (CMO) one or more of the following test results: i) positive test for anti-TBEV IgM serum antibodies, ii) anti-TBEV IgG serum antibody seroconversion, iii) a $\geq 4$-fold rise in anti-TBEV IgG serum antibodies in paired serum specimens, and iv) successful TBEV genome 
TABLE 1

Classification of reported tick-borne encephalitis cases, Switzerland, 2005-2011

\begin{tabular}{|l|l|l|l|}
\hline Case classification & \multicolumn{2}{|l|}{ Laboratory criteria } & Clinical criteria \\
\hline \multirow{2}{*}{ Not a case } & \multicolumn{2}{|l|}{ Positive IgM serology } & No ILI and no neurological symptoms \\
\hline \multirow{2}{*}{ Probable case } & a) & Positive IgM serology & ILI or non-specific neurological signs and symptoms \\
\hline & b) & Positive IgM + positive IgG serology & Any \\
\hline \multirow{2}{*}{ Confirmed case } & a) & Positive IgM serology & Meningitis, meningoencepalitis, encephalomyelitis or pareses \\
\hline & b) & Positive IgM + positive IgG serology & ILI or non-specific neurological signs and symptoms \\
\hline & a) & Positive IgM + positive IgG serology & Meningitis, meningoencepalitis, encephalomyelitis or pareses \\
\hline & b) & TBE-RNA detection by PCR & Meningitis, meningoencepalitis, encephalomyelitis or pareses \\
\hline
\end{tabular}

ILI: influenza-like illness; TBE: tick-borne encephalitis.

a or anti-TBE IgG serum antibody seroconversion or $\geq 4$-fold rise in anti-TBE IgG serum antibodies.

amplification. The CMO is then obliged to ask for additional information from the patient's physician, such as clinical presentation, disease progression, TBE vaccination history and tick bite within four weeks before onset of illness, in a standardised reporting form and submit this to the FOPH [9]. All data for this study have been provided by the FOPH in compliance with Swiss data protection laws.

\section{Data inclusion and surveillance definitions}

Patients were excluded from further analysis if they were classified as 'not a case' on the basis of laboratory results and documented clinical characteristics, whereas possible, probable and confirmed cases were included for further analysis (Table 1 ). The reason to include possible, probable and confirmed cases into the analysis was to monitor TBE infection rather than only the disease, and to compare time trends with the past data from 1984-2004 [4]. Patients resident in the neighbouring Principality of Liechtenstein (FL) were excluded.

\section{Analysis}

The data set for analysis comprises possible, probable and confirmed cases according to the definitions given in Table 1. All analysis of data was done using Microsoft Excel 2008 and SPSS (version 20.0, SPSS, Inc., Chicago, United States) software. Analysis included cross tables and incidence estimations. No statistical inference was done because surveillance data are not a random sample.

\section{FIGURE 1}

Annual incidence of tick-norne encephalitis in Switzerland, 1984 to $2011(n=2,421)$

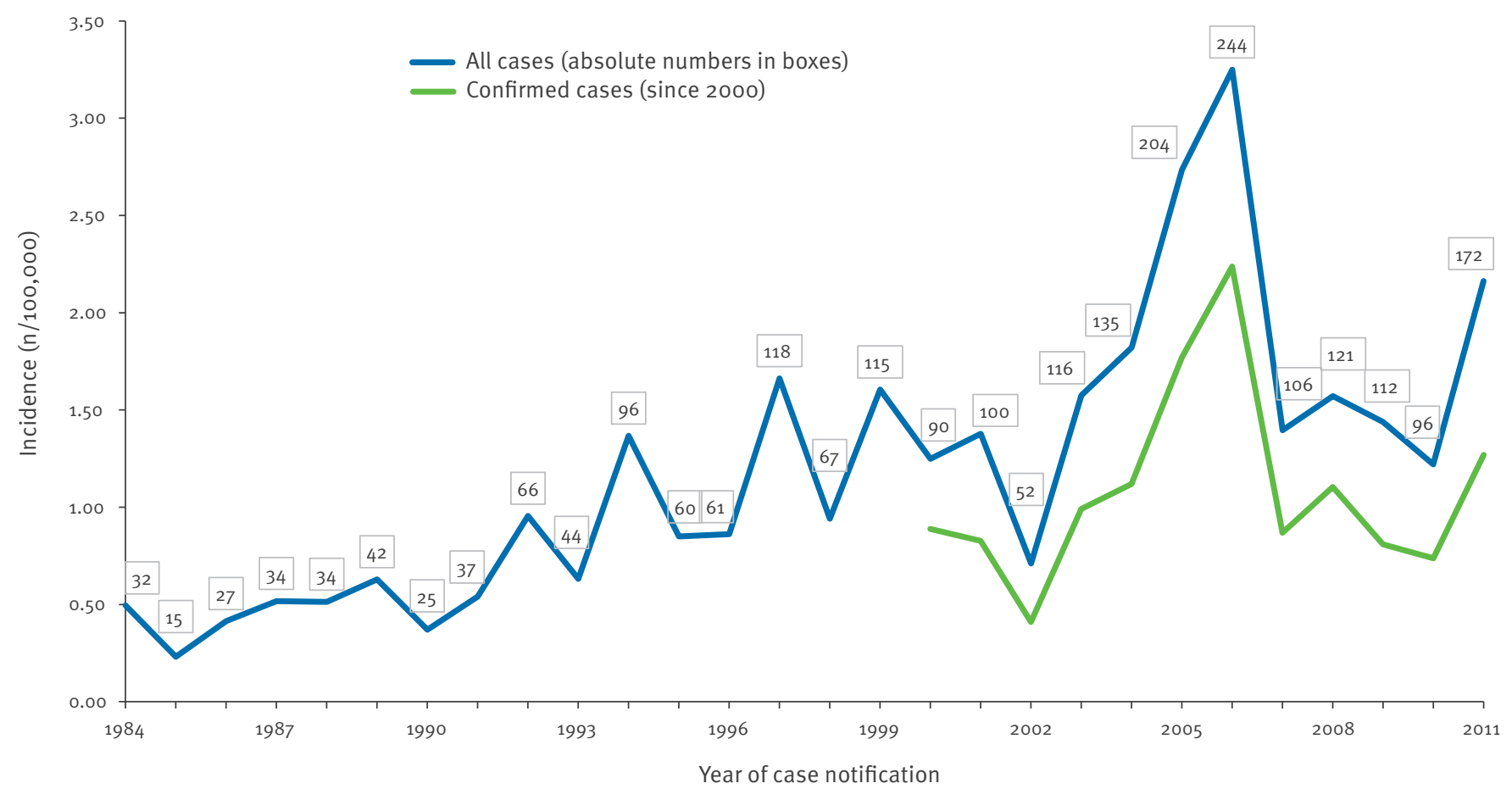


TABLE 2

Number and incidence per 100,000 inhabitants of tick-borne encephalitis cases by year and canton of residence, Switzerland, 2005 to $2011(n=1,055)$

\begin{tabular}{|c|c|c|c|c|c|c|c|c|}
\hline \multirow{2}{*}{ Canton } & \multicolumn{8}{|c|}{ Yearly incidence (total number) } \\
\hline & 2005 & 2006 & 2007 & 2008 & 2009 & 2010 & 2011 & $2005-2011$ \\
\hline TG & $9.8(23)$ & $9.3(22)$ & $8.0(19)$ & $3.7(9)$ & $10.2(25)$ & $6.4(16)$ & 7.5 (19) & $7.8(133)$ \\
\hline NW & $7.5(3)$ & $20.0(8)$ & $7.4(3)$ & $2.5(1)$ & $2.5(1)$ & $0.0(0)$ & $12.1(5)$ & $7.4(21)$ \\
\hline UR & $2.9(1)$ & $11.4(4)$ & $8.6(3)$ & $8.5(3)$ & $0.0(0)$ & $2.8(1)$ & $0.0(0)$ & $4.9(12)$ \\
\hline AG & $6.3(36)$ & $4.7(27)$ & $2.2(13)$ & $2.2(13)$ & $2.2(13)$ & $1.8(11)$ & $3.7(23)$ & $3.3(136)$ \\
\hline $\mathrm{ZH}$ & $4.9(62)$ & $5.4(69)$ & $2.4(32)$ & $3.3(44)$ & $2.2(30)$ & $1.4(19)$ & $3.1(43)$ & $3.2(299)$ \\
\hline LU & $5.3(19)$ & $5.3(19)$ & $1.7(6)$ & $1.6(6)$ & $1.6(6)$ & $2.4(9)$ & $2.4(9)$ & $2.9(74)$ \\
\hline $\mathrm{Al}$ & $0.0(0)$ & $6.5(1)$ & $0.0(0)$ & $6.4(1)$ & $0.0(0)$ & $0.0(0)$ & 6.4 (1) & $2.8(3)$ \\
\hline $\mathrm{SH}$ & $5.4(4)$ & $4.1(3)$ & $0.0(0)$ & $2.7(2)$ & $2.6(2)$ & $2.6(2)$ & $1.3(1)$ & $2.7(14)$ \\
\hline $\mathrm{BE}$ & $1.6(15)$ & $4.2(40)$ & $0.6(6)$ & $2.1(20)$ & $1.7(17)$ & $1.3(13)$ & $2.2(22)$ & 2.0 (133) \\
\hline SG & 2.6 (12) & $1.1(5)$ & $1.5(7)$ & $1.7(8)$ & $1.5(7)$ & $1.7(8)$ & $3.3(16)$ & $1.9(63)$ \\
\hline OW & $6.0(2)$ & $3.0(1)$ & $0.0(0)$ & $2.9(1)$ & $0.0(0)$ & $0.0(0)$ & $0.0(0)$ & $1.7(4)$ \\
\hline GR & $3.2(6)$ & $2.7(5)$ & $1.6(3)$ & $1.1(2)$ & $1.6(3)$ & $0.5(1)$ & $1.0(2)$ & $1.7(22)$ \\
\hline$A R$ & $1.9(1)$ & $1.9(1)$ & $0.0(0)$ & $0.0(0)$ & $3.8(2)$ & $0.0(0)$ & $3.8(2)$ & $1.6(6)$ \\
\hline FR & $1.2(3)$ & 3.9 (10) & $1.1(3)$ & $0.4(1)$ & $1.1(3)$ & $1.4(4)$ & $2.1(6)$ & $1.6(30)$ \\
\hline $\mathrm{GL}$ & $2.6(1)$ & $2.6(1)$ & $0.0(0)$ & $0.0(0)$ & $0.0(0)$ & $2.6(1)$ & $0.0(0)$ & $1.1(3)$ \\
\hline So & $2.0(5)$ & $2.4(6)$ & $0.4(1)$ & $0.0(0)$ & $0.0(0)$ & $0.4(1)$ & $0.8(2)$ & 0.9 (15) \\
\hline $\mathrm{SZ}$ & $0.7(1)$ & $0.0(0)$ & $1.4(2)$ & $0.0(0)$ & $0.0(0)$ & $1.4(2)$ & $2.0(3)$ & $0.8(8)$ \\
\hline VD & $0.8(5)$ & 1.7 (11) & $0.7(5)$ & $0.9(6)$ & $0.1(1)$ & $0.3(2)$ & $1.1(8)$ & $0.8(38)$ \\
\hline BS & $0.5(1)$ & $2.2(4)$ & $0.0(0)$ & $0.5(1)$ & $0.0(0)$ & $0.5(1)$ & $1.1(2)$ & 0.7 (9) \\
\hline JU & $1.4(1)$ & $1.4(1)$ & $0.0(0)$ & $0.0(0)$ & $0.0(0)$ & $1.4(1)$ & $0.0(0)$ & $0.6(3)$ \\
\hline $\mathrm{BL}$ & $0.8(2)$ & $1.1(3)$ & $0.7(2)$ & $0.0(0)$ & $0.4(1)$ & $0.7(2)$ & $0.4(1)$ & 0.6 (11) \\
\hline$Z G$ & $0.9(1)$ & $0.0(0)$ & $0.0(0)$ & $0.9(1)$ & $0.9(1)$ & $0.0(0)$ & $0.9(1)$ & $0.5(4)$ \\
\hline VS & $0.0(0)$ & $0.3(1)$ & $0.0(0)$ & $0.3(1)$ & $0.0(0)$ & $0.6(2)$ & $1.3(4)$ & $0.4(8)$ \\
\hline $\mathrm{NE}$ & $0.0(0)$ & $1.2(2)$ & $0.0(0)$ & $0.0(0)$ & $0.0(0)$ & $0.0(0)$ & $0.6(1)$ & $0.3(3)$ \\
\hline $\mathrm{TI}$ & $0.0(0)$ & $0.0(0)$ & $0.0(0)$ & $0.3(1)$ & $0.0(0)$ & $0.0(0)$ & $0.3(1)$ & $0.1(2)$ \\
\hline GE & $0.0(0)$ & $0.0(0)$ & $0.2(1)$ & $0.0(0)$ & $0.0(0)$ & $0.0(0)$ & $0.0(0)$ & $0.1(1)$ \\
\hline $\mathrm{CH}$ & $2.7(204)$ & $3.3(244)$ & $1.4(106)$ & $1.6(121)$ & $1.4(112)$ & $1.2(96)$ & $2.2(172)$ & $2.0(1,055)$ \\
\hline
\end{tabular}

AG: Aargau; Al: Appenzell Innerrhoden; AR: Appenzell Ausserrhoden; BE: Bern; BL: Basel (county); BS: Basel (city); CH: Switzerland; FR: Fribourg; GE: Geneva; GL: Glarus; GR: Graubünden; JU: Jura; LU: Lucerne; NE: Neuchâtel; NW: Nidwalden; OW: Obwalden; SG: Saint Gallen; SH: Schaffhausen; SO: Solothurn; SZ: Schwyz; TG: Thurgau; TI: Ticino; UR: Uri; VD: Vaud; VS: Valais; ZG: Zug; ZH: Zurich.

TBE incidences were calculated based on data on the permanent resident population provided by the Federal Statistical Office [10]. Incidence rates by canton refer to the patient's place of residence, not to the presumed place of a tick bite.

\section{Results}

\section{General characteristics}

Between 2005 and 2011, 1,177 reports of TBE were notified to the FOPH. Of these, 114 (9.7\%) were categorised as 'not a case' and excluded, as were the eight patients (0.7\%) living in FL. Of the remaining 1,055 cases, laboratory reports were available for 1,004 (95\%), and the physician reporting form with clinical and laboratory data was available for 1,008 (96\%). For 47 cases, only the laboratory report was available. Complete data sets were available from 1,001 patients (95\%). Of the 1,055 cases, $133(13 \%)$ were classified as possible cases, 249 (24\%) as probable cases and $673(64 \%)$ as confirmed cases.

\section{Epidemiological findings}

There were two peaks of reported TBE cases (combining possible, probable and confirmed cases as defined in Table 1) during the study period: one during the years $2005(n=204)$ and $2006(n=244)$ and one in 2011 $(n=172)$. From 2007 to 2010 , the number of cases was relatively stable with an average of 109 cases per year.

The yearly national incidence rates (n cases/100,000 inhabitants) in Switzerland fluctuated within a range of 1.2 (2010) and 3.3 (2006), leading to an average yearly incidence of 2.0 for the whole study period, with the highest average value of 7.8 in the canton of Thurgau in eastern Switzerland (Figure 1, Table 2). When the incidence of reported TBE cases since initiation of surveillance in 1984 is considered (Figure 1), an increasing 


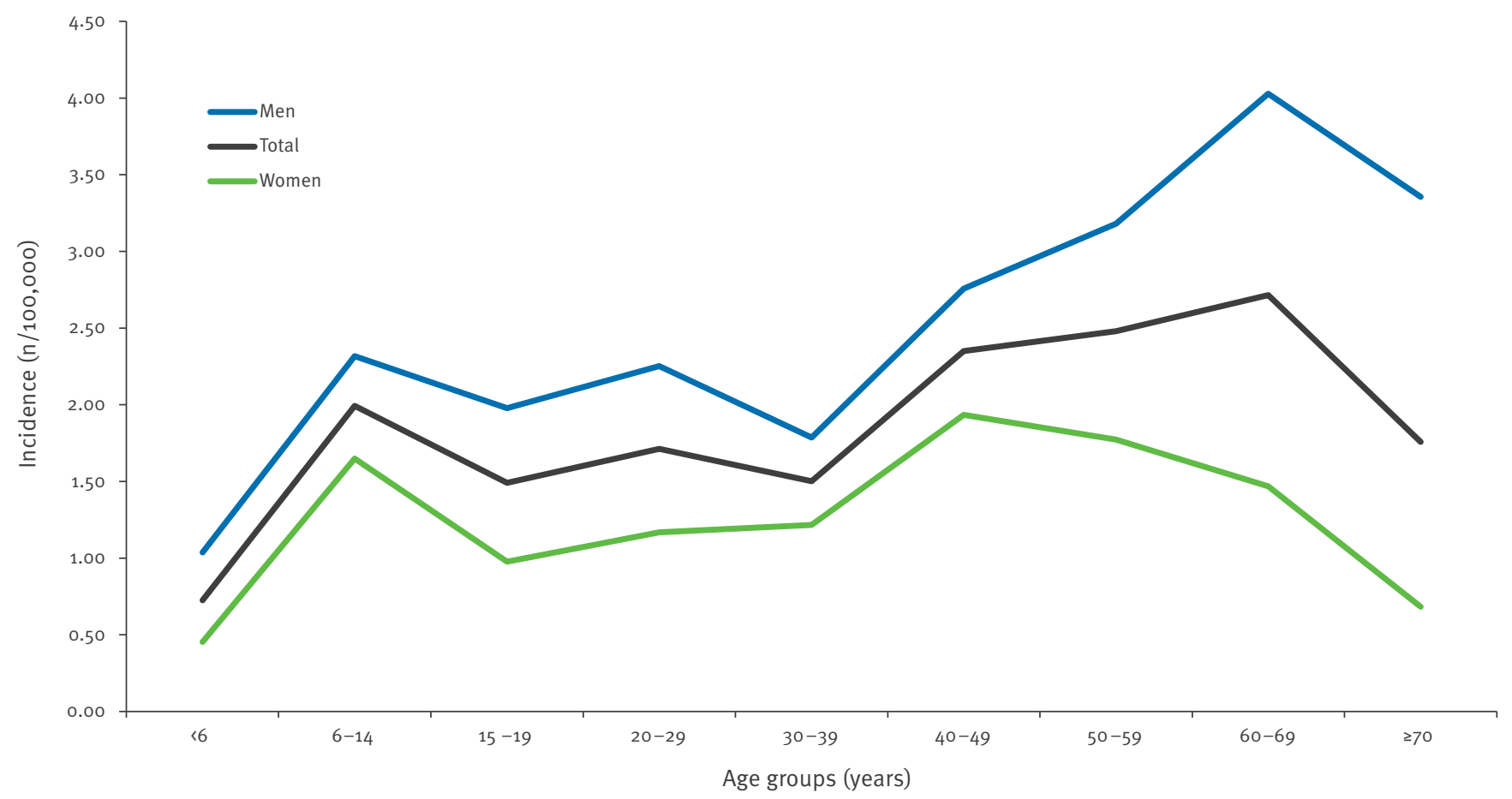

trend of yearly incidences can be seen. Each of the 26 Swiss cantons reported at least one patient with TBEV infection during the study period. Nonetheless, case numbers fluctuated considerably from year to year, with only nine cantons notifying at least one patient every year.

Incidence was consistently higher in men in all age groups (Figure 2). Further, incidence was low in children under the age of six years, constant at equal levels for age groups between six and 39 years, and reached highest values between 40 and 69 years of age.

Most cases of TBE with known date of manifestation ( $n=884$ ) occurred between April and September, peaking in June $(n=221 ; 25 \%)$ and July $(n=228 ; 26 \%)$. From October to March, only few cases with TBE were reported: three $(0.3 \%$ of total) in January, one (0.1\%) in February, eight (0.9\%) in March and 10 (1.1\%) in December. Comparison of different age groups showed no substantial variability with regards to TBE seasonality (data not shown).

In 478 (45\%) of 1,055 patients, a history of tick bite(s) within four weeks before onset of illness was reported, whereas 147 patients (14\%) could not remember any tick bite. In 430 patients ( $41 \%)$ the history of tick bite was unknown. Sixty-seven (6.4\%) patients experienced tick bites outside their cantons of residence, 50 (4.7\%) in Switzerland and $17(1.6 \%)$ in a foreign country. Almost half of the patients $(n=502 ; 48 \%)$ were either bitten in the canton of their residence or had permanently stayed in the canton of their residence during the maximal incubation period, i.e. up to four weeks before onset of illness.

\section{Clinical findings}

Meningoencephalitis was the most frequent manifestation in probable and confirmed TBE cases and overall (Figure 3). A clinical diagnosis was available in 1,001 of 1,008 cases $(99 \%)$ with physician report. The official TBE notification form requires reporting physicians to specify the patient's CNS manifestations as one of five clinical diagnoses: 'meningitis', 'meningoencephalitis', 'encephalomyelitis', 'radiculitis' and 'others' where free text can be entered [9]. Due to the difficulties in exact clinical differentiation and according to international literature $[1,2,11]$, we combined encephalomyelitis and radiculitis in one category for the purposes of our analysis. Furthermore, patients presenting with headache, vertigo, paraesthesia, myoclonia and similar manifestations as reported under 'others' were categorised together as 'non-specific neurological signs and symptoms'. Five patients received the diagnosis 'paresis' without indication of other neurological symptoms (i.e. no meningitis, meningoencephalitis or radiculitis): three had a paresis of the facial nerve, one had an unspecified cranial nerve paresis and one had a paresis of unknown localisation. The total number of patients with paresis (combined with other neurological manifestations) is unknown. Of 617 patients of at least 40 years of age, $442(72 \%)$ suffered from a severe course of illness, i.e. with meningoencephalitis, encephalomyelitis or pareses, compared with 196 $(51 \%)$ of 384 patients under the age of 40 years (Figure 
Incidence of clinical findings in cases of tick-born encephalitis, by age, Switzerland, 2005 to $2011(\mathrm{n}=1,001)^{\star}$

A: Confirmed TBE cases $(n=673)$

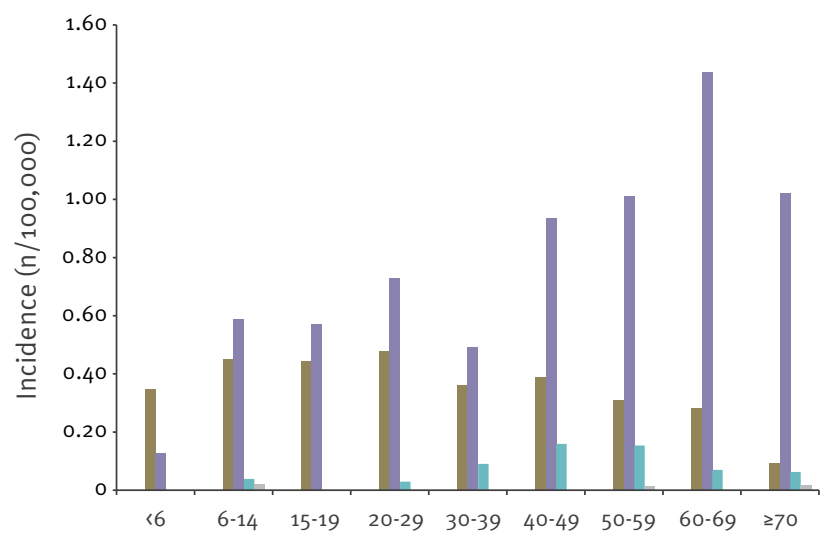

B: Probable TBE cases $(n=249)$

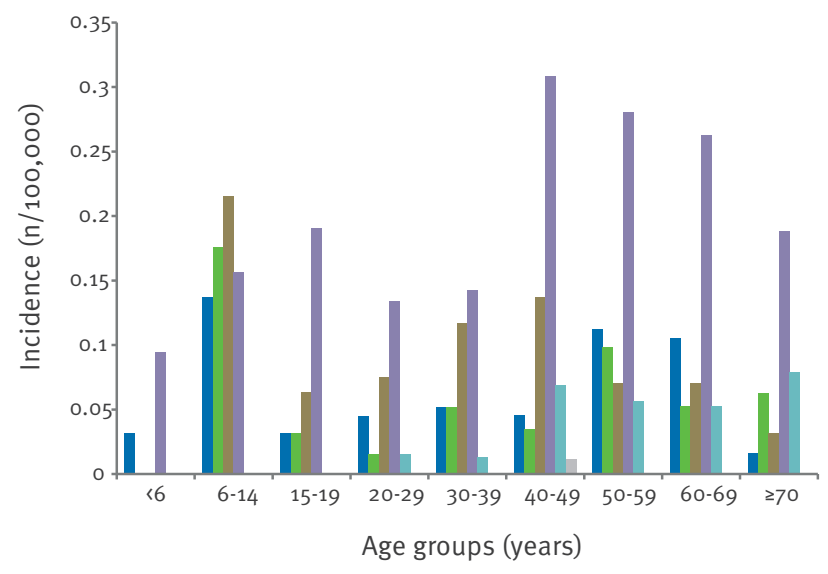

Non-specific neurological signs or symptoms
C: PossibletBE cases $(n=79)$

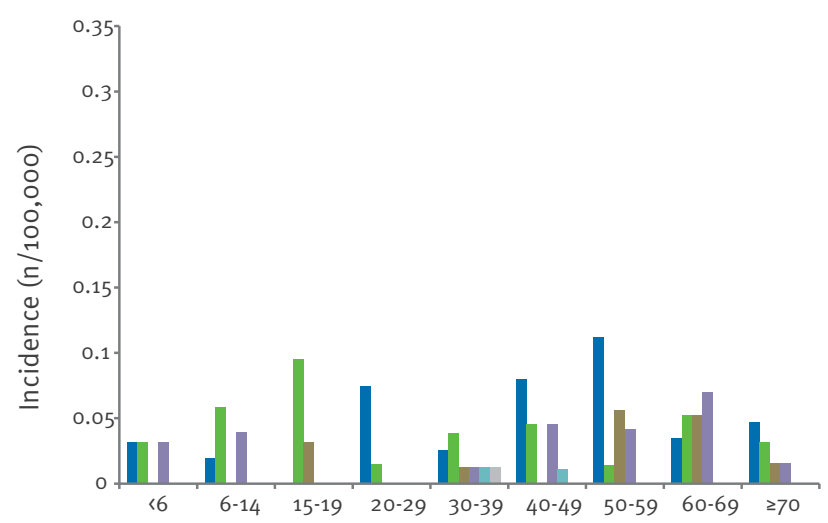

D: All TBE cases $(n=1,001)$

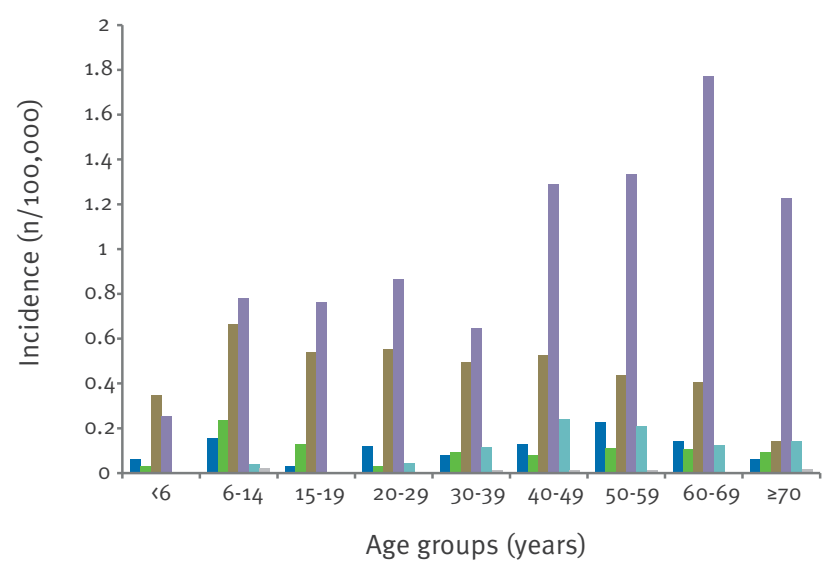

Encephalomyelitis Unknown

ILI: influenza-like illness; TBE: tick-borne encephalitis

3). There was no year-by-year variability in proportions of diagnoses from 2005 to 2011 (data not shown).

Of the 1,055 patients with TBE, 789 (75\%) were hospitalised, 193 (18\%) were reportedly treated as outpatients, and for $73(6.9 \%)$ patients, the respective information was missing. Precise dates of admission to and discharge from hospital were available for 666 ( $84 \%$ ) of the 789 hospitalised patients. The mean duration of hospitalisation was 9.0 days (interquartile range (IQR): 5-11 days). Duration of hospitalisation increased with the age of the patient in a linear pattern, from a mean of 5.0 days (IQR: 3-7 days) in children up to the age of 14 years to a maximum mean of 14.6 days (IQR: 9-18 days) in those 70 years and older.

Nine (0.9\%) of 1,055 patients died in timely association with TBE. For seven of them, the course of the TBE illness was fatal, whereas the remaining two deaths were most likely unrelated to TBE: one patient died after abdominal surgery and another after a bicycle accident. Of the seven patients with fatal TBE, five had a diagnosis of meningoencephalitis and two had a diagnosis of encephalomyelitis. The age range of these seven patients was 15 to 87 years (median: 81 years), three were female (age: 15-38 years), four were male (age: $81-87$ years).

Tick-borne encephalitis immunisation history Sixty-five $(7.9 \%)$ of 822 patients with known immunisation status had a history of at least one dose of TBE vaccine administered at least four weeks before onset of disease, and 38 (4.6\%) patients had received a complete primary vaccination series with at least three doses (Figure 4). In 19 of these 38 patients, the last dose was administered less than three years before disease onset. In five patients (three confirmed, one probable and one possible case), the last dose had 
FigURE 4

Vaccination history in patients with reported tick-borne encephalitis in Switzerland, 2005 to $2011(n=1,055)$

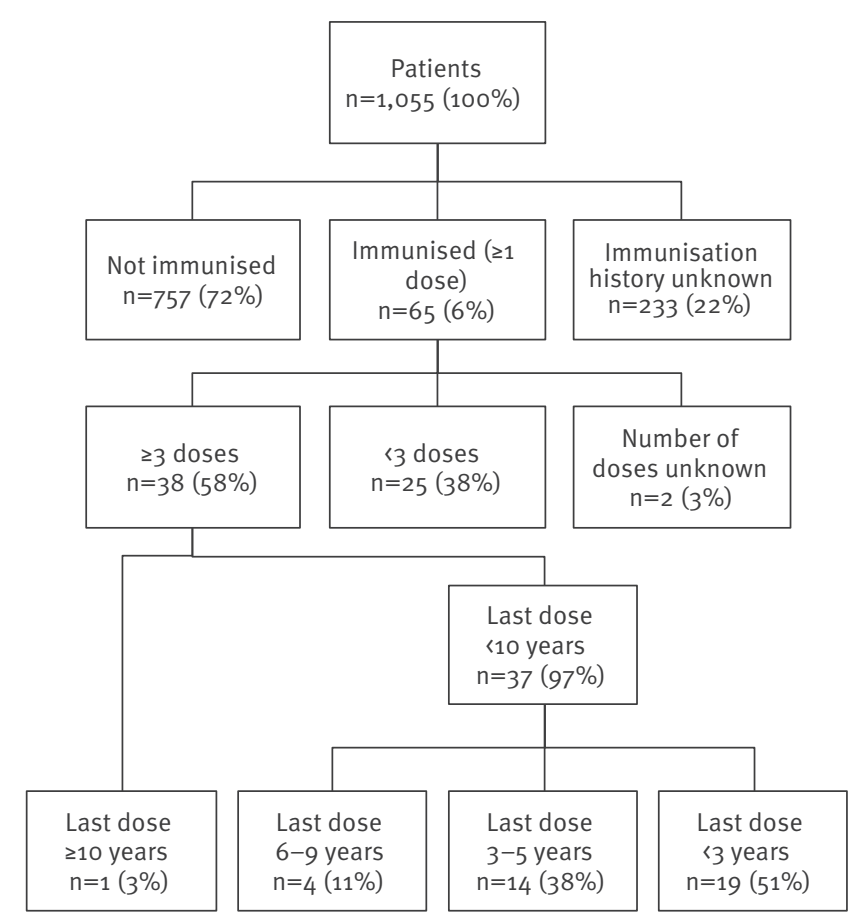

been administered more than five years before TBE disease; it is therefore possible that the disease in these five patients could have been prevented with a booster dose, as indicated in the package insert approved by Swissmedic $[12,13]$. As the coverage of vaccinated people in Switzerland is not known, it is not possible to calculate whether these rare cases are in the limits of expected vaccine failures. Of the remaining 33 patients $(4.0 \%)$ with possible vaccine failure (i.e. who had received at least three doses of vaccine and the last dose within five years before disease onset), 19 patients $(2.3 \%$ of 822$)$ were classified as confirmed, three (0.4\%) as probable and $11(1.3 \%)$ as possible cases.

The median age of patients with completed vaccination series with at least three doses $(n=38)$ was 53 years (IQR: $37-65.5$ years), that of unimmunised patients $(n=757)$ was 46 years (IQR: $27-59$ years). The frequency of hospitalisation in patients with at least three doses of TBE vaccine was lower ( 26 of $38,68 \%$ ) than that of unimmunised patients (592 of $757,78 \%$ ).

\section{Discussion}

Between 2005 and 2011, a total of 1,055 TBE cases fulfilling the case definition and living in Switzerland were reported to the $\mathrm{FOPH}$, with a peak of 244 cases in 2006. When compared to the previous surveillance period, 1984 to 2004 [4], the average annual incidence increased from 1.4 to 2.0 in this current study. This could be due to an increase in endemic areas, in the tick population, or in human exposure through increased outdoor activities, or a combination of these factors. Similar trends have been observed in several other TBE-endemic countries in Europe [14]. Alternatively, increased awareness of TBE may have lead to increased reporting.

Still, the presented data on TBE in Switzerland are probably underestimates because of missed diagnoses and possible underreporting. If the physician does not suspect TBE, a laboratory test for TBE will not be ordered and therefore the diagnosis will be missed. This is particularly possible assuming that meningitis or ILI are the leading clinical features. In agreement with this assumption, fewer cases of TBE meningitis than cases of TBE meningoencephalitis are reported in Switzerland, which is in contrast to reports from other European countries with endemic TBE $[1,11]$.

In comparison to other TBE-endemic countries in Europe, the incidence in Switzerland (range 1.2$3.3 / 100,000$ ) is low. Especially in eastern Europe, much higher yearly incidences were reported for the period 2005 to 2009, such as those from the Czech Republic (range: 5.3-10.0/100,000), Estonia (range: 6.7-13.3/100,000), Latvia (range: 6.2-14.6/100,000) or Slovenia (range: 9.9-18.6/100,000) [14]. In contrast, the nationwide average incidence in Germany in 2005 to 2011 (range: 0.3-0.7/100,000) was lower than in Switzerland, but values in the endemic regions of southern Germany, e.g. Baden-Württemberg (range: 0.9-2.6/100,000) and Bavaria (range: 0.8-1.7/100,000) were comparable to those of neighbouring Switzerland [15]. Until the national mass vaccination campaign was started in 1981, Austria was the country with the highest recorded TBE morbidity in Europe, with incidences ranging from 3.9 to 9.0 per 100,000 in the period from 1972 to $1982[16,17]$. Not surprisingly, reported incidence rates in the non-immunised Austrian population are still in this range, whereas they dropped to about 1 per 100,000 (range for 2005-2011: 0.6-1.3) in the total population due to a vaccination coverage of at least $85 \%$ which was achieved during the past decade [17-19].

Epidemiological data from different countries should be compared with caution, as there are different reporting standards, varying case definitions and classifications as well as differences in vaccination coverage. In an effort to improve data comparability in the future, the European Commission proposed an institutional case definition for EU Member States in August 2012 [20]. In contrast to our current case classification, the new classification only differentiates between probable and confirmed cases, but does not define possible cases. Furthermore, only manifestations of CNS inflammation such as meningitis, meningoencephalitis, encephalomyelitis and encephaloradiculitis are accepted clinical TBE criteria, whereas ILI or non-specific neurological signs and symptoms do not qualify a case. Still, $81 \%$ (673 confirmed and 182 probable cases, Figure 3, panels $A$ and $B$ ) of our 1,055 cases fulfilled the new EU case 
definitions. It is of importance that not only disease is monitored, but also infection, because infection is a much more sensitive indicator than disease with respect to the epidemiology.

TBE is a disease that occurs in local endemic regions, therefore population-based incidence rates have to be interpreted cautiously [21]. Because history of tick bite was reported by less than half of the patients in this study, we decided to determine incidence rates based on the patients' cantons of residence. Local incidence rates can be much higher than the reported national average of 2 per 100,000 inhabitants; they can also be higher than incidences by canton because endemic foci do not match with political borders.

Despite convincing evidence of the vaccine's effectiveness and safety [22], TBE vaccination coverage in endemic regions of Switzerland is in the range of approximately $25-50 \%$ and therefore still low [7]. In our study population, most of the patients were not immunised against TBEV or their immunisation history was unknown ( $n=990 ; 94 \%)$. Assuming a 99\% effectiveness of complete vaccination [5], TBE could have been prevented in 1,041 of the 1,055 patients. Nevertheless, $3.1 \%$ of the patients were completely immunised (at least three doses, last dose within five years before disease) and have to be regarded as cases of apparent vaccine failure. Considering that no curative treatment of TBE exists and that there is high long-term morbidity [1], more effort should be made to increase TBE vaccination coverage in risk groups.

In conclusion, ongoing surveillance of TBE and intensified efforts in promoting TBE vaccination for the population at risk will be needed in light of the increasing incidence and its considerable morbidity.

\section{*Author's correction:}

On request of the authors, subtitles in Figure 3 as well as the graph in Figure 3A were corrected on 7 April 2014.

\section{Conflict of interest}

None declared.

\section{Authors' contributions}

HpZ has collected the TBE data. MS, HpZ and UH have analysed the data. MS wrote the first draft of the manuscript. All other authors have contributed to further versions of the manuscript and approved the final version before submission.

\section{References}

1. Kaiser R. The clinical and epidemiological profile of tick-borne encephalitis in southern Germany 1994-98: a prospective study of 656 patients. Brain. 1999;122(Pt11):2067-78. http://dx.doi.org/10.1093/brain/122.11.2067
2. Dumpis U, Crook D, Oksi J. Tick-borne encephalitis. Clin Infect Dis. 1999;28(4):882-90.

http://dx.doi.org/10.1086/515195

3. Haglund M, Günther G. Tick-borne encephalitis - pathogenesis, clinical course and long-term follow-up. Vaccine. 2003;21 Supp $1:$ S $11-8$.

http://dx.doi.org/10.1016/S0264-410X(02)00811-3

4. Zimmermann H, Koch D. [Epidemiology of tick-borne encephalitis (TBE) in Switzerland 1984 to 2004]. Ther Umsch. 2005;62(11):71925. German.

http://dx.doi.org/10.1024/0040-5930.62.11.719

5. Heinz FX, Holzmann H, Essl A, Kundi M. Field effectiveness of vaccination against tick-borne encephalitis. Vaccine. 2007;25(43):7559-67. http://dx.doi.org/10.1016/j. vaccine.2007.08.024

6. Kunze U. TBE - awareness and protection: the impact of epidemiology, changing lifestyle, and environmental factors. Wien Med Wochenschr. 2010;160(9-10):252-5 http://dx.doi.org/10.1007/s10354-010-0798-x

7. Tabelle mit vollständigen Resultaten zur Durchimpfung 1999-2012. [Table with complete results on Vaccination coverage 1999-2012]. Berne: Bundesamt für Gesundheit. [Accessed: 21 Mar 2013]. German. Available from: http://www.bag.admin.ch/themen/ medizin/o0682/00685/02133/index.html?lang=de

8. Bundesamt für Gesundheit. Zeckenenzephalitis (FSME): weitere Zunahme der gemeldeten Fälle im 2006. [Tick-borne encephalitis (TBE): further increases in reports cases in 2006]. Bulletin BAG. 2007;4:57-60. German.

9. Meldeformular Zeckenenzepahlitis. [Reporting form tick-borne encephalitis]. Berne: Bundesamt für Gesundheit. [Accessed: 31 Jan 2013]. German. Available from: http://www.bag.admin.ch/k_m_ meldesystem/00733/00814/index.html?lang=de

10. Bevölkerungsstand und -struktur - Detaillierte Daten. [Population status and structure - detailed data]. Neuchâtel: Bundesamt für Statistik. [Accessed: 31 Jan 2013]. German. Available from: http:// www.bfs.admin.ch/bfs/portal/de/index/themen/01/02/blank data/o1.html

11. Karelis G, Bormane A, Logina I, Lucenko I, Suna N, Krumina A, et al. Tick-borne encephalitis in Latvia 1973-2009: epidemiology, clinical features and sequelae. Eur I Neurol. 2012;19(1):62-8. http://dx.doi.org/10.1111/j.1468-1331.2011.03434.x

12. Baxter AG. FSME-Immun - Fachinformation des Arzneimittelkompendium Schweiz. [FSME-Immun - professional information of the drug compendium Switzerland]. Basel: Documed AG. [Accessed 18 Feb 2013]. German. Available from: http:// compendium.ch/mpro/mnr/1932/html/de

13. Novartis Pharma. Encepur $\mathrm{N}$ - Fachinformation des Arzneimittelkompendium Schweiz. [Encepur N - professional information of the drug compendium Switzerland]. Basel: Documed AG. [Accessed $18 \mathrm{Feb}$ 2013]. Available from: http://compendium.ch/ $\mathrm{mpro} / \mathrm{mnr} / 9151 / \mathrm{html} / \mathrm{de}$

14. Donoso Mantke O, Escadafal C, Niedrig M, Pfeffer M, on behalf of the Working group for Tick-borne encephalitis virus. Tickborne encephalitis in Europe, 2007 to 2009. Euro Surveill. 2011;16(39): $\mathrm{pii}=19976$.

15. Robert Koch Institute (RKI). SurvStat. Berlin: RKI. [Accessed 20 Feb 2013]. German. Available from: http://www3.rki.de/SurvStat

16. Kunz C. TBE vaccination and the Austrian experience. Vaccine. 2003;21(Suppl 1):S50-5.

http://dx.doi.org/10.1016/S0264-410X(02)00813-7

17. Heinz FX, Stiasny K, Holzmann H, Grgic-Vitek M, Kriz B, Essl A, et al. Vaccination and Tick-borne Encephalitis, Central Europe. Emerg Infect Dis. 2013:19(1):69-76 http://dx.doi.org/10.3201/eid1901.120458

18. Clinical Institute of Virology. Virusepidemiologische Information 2006-2012. [Virus epidemiological information 2006-2012]. Vienna: Medical University of Vienna. [Accessed: 21 Feb 2013]. German. Available from: http://www.virologie.meduniwien.ac.at/ home/virus-epidemiologie/virusepidemiologische-information/ lang_1-content.html

19. Statistik Austria. Bevölkerungsstand und Bevölkerungsveränderung. [Population status and population change]. Vienna: Bundesanstalt Statistik Österreich. [Accessed: 5 Mar 2013]. German. Available from: http://www.statistik.at/ web de/statistiken/bevoelkerung/bevoelkerungsstand und veraenderung/index.html

20. European Commission. Commission Implementing Decision of 8 August 2012 amending Decision 2002/253/EC laying down case definitions for reporting communicable diseases to the Community network under Decision No 2119/98/EC of the European Parliament and of the Council (2012/506/EU). Official Journal of the European Union. Luxembourg: Publications Office of the European Union; 27.9.2012:L262. Available from: http://eur-lex.europa.eu/JOHtml. 27.9.2012:L262. Available from: http:

21. Zeckenenzephalitis - Verbreitung der Endemiegebiete. [Tick-borne encephalitis - distribution of endemic areas]. Berne: Bundesamt für Gesundheit. [Accessed: 31 Jan 2013]. German. Available from: http://map.geo.admin.ch/? $X=190000.00 \& Y=660000.00 \& z 00$ $\mathrm{m}=1 \&$ lang=en\&topic $=$ ech \&bgLayer $=$ ch. swisstopo. pixelkarte farbe\&layers =ch.bag.zecken-fsme-impfung\&layers_opacity $=0.75$

22. Schumacher Z, Bourquin C, Heininger U. Surveillance for adverse events following immunization (AEFI) in Switzerland - 1991-2001. Vaccine. 2010;28(24):4059-64. http://dx.doi.org/10.1016/j.vaccine.2010.04.002 